\title{
METODOLOGIAS DE DETERMINAÇÃO DO VOLUME DE MADEIRA EM PÉ PARA APLICAÇÃO EM INVENTÁRIOS FLORESTAIS
}

Plínio Carielo, Renato de Araújo Ferreira, Bruno Vicente de Amaral, Emerson Ferreira de Oliveira, João Pedro de Freitas Melo, Vinicius Alves Dias ${ }^{6}$

Escola Técnica Estadual Prof. Dr. Antonio Eufrásio de Toledo, curso Técnico em Florestas, Presidente Prudente - SP. Email: pliniocarielo@hotmail.com

\section{RESUMO}

Os inventários florestais consistem em utilizar técnicas de amostragem para obtenção de dados quantitativos e qualitativos de povoamentos florestais. O volume constitui uma das informações de maior importância para o conhecimento do potencial disponível em um povoamento florestal. Existem diversas metodologias de inventários florestais, entretanto, para todas as metodologias é necessário realizar o procedimento de cubagem rigorosa em árvores representativas da floresta para posteriormente determinar o fator de forma médio, o fator razão ou a equação volumétrica que permitira a determinação do volume em pé de todo o povoamento florestal. Dentre as metodologias as equações volumétricas são as melhores e mais recomendadas.

Palavras-chave: Fator de Forma Médio, Fator Razão, Equações Volumétricas, Regressão, Cubagem Rigorosa.

\section{METHODOLOGIES FOR DETERMINING THE VOLUME OF WOOD STANDING FOR APPLICATION IN FOREST INVENTORY}

\begin{abstract}
The forest inventories consist of using sampling techniques to obtain quantitative and qualitative data of forest stands. The volume is one of the most important information for understanding the potential available in a forest stand. There are several methodologies for forest inventories, however, for all methodologies is necessary to perform the procedure cubed representative trees in the forest to subsequently determine the mean form factor, the factor ratio or volumetric equation that allowed the determination of standing volume the entire forest stand. Among the methodologies volumetric equations are the best and most recommended.
\end{abstract}

Keywords: Medium Form Factor, Factor Ratio, Volumetric Equations, Regression, Cubic Rigorous.

\section{INTRODUÇÃO}

Os inventários florestais consistem em utilizar técnicas de amostragem para obtenção de dados quantitativos e qualitativos de povoamentos florestais a fim de se determinar o volume de madeira presente e/ou futuro de uma floresta.

O volume constitui uma das informações de maior importância para o conhecimento do potencial disponível em um povoamento florestal, haja vista que o volume individual fornece 
subsídios para a avaliação do estoque de madeira e análise do potencial produtivo das florestas (THOMAS et al., 2006).

Existem diversas metodologias de inventários florestais das mais simples as mais elaboradas que são utilizadas conforme o tipo de floresta e os objetivos que se pretende obter com a realização do mesmo. Para todas as metodologias é necessário realizar o procedimento de cubagem rigorosa em árvores representativas da floresta. Este procedimento permite determinar o volume real em metros cúbicos de cada uma das árvores cubadas, para posteriormente determinar o fator de forma médio, o fator razão ou a equação volumétrica que permitirá posteriormente determinar o volume de toda a população.

Diante de tal fato, este trabalho comparou os volumes obtidos para as parcelas de um inventário florestal de uma floresta clonal de Eucalyptus sp. através da aplicação das diferentes metodologias de determinação do volume de madeira de árvores em pé: Fator de Forma Médio, Fator Razão, e Equação Volumétrica.

\section{METODOLOGIA}

\section{Caracterização do Local}

Os dados utilizados foram coletados durante a realização de um inventário florestal tipo convencional de um povoamento clonal do gênero Eucalyptus, clone GG100, com uma área de 548,8 hectares, com quatro anos de idade, plantados no espaçamento $3 \times 2 \mathrm{~m}$, no município de Água Clara - MS.

A amostra definitiva utilizada no inventário florestal foi de 36 parcelas retangulares, $30 \mathrm{x}$ $24 \mathrm{~m}$, totalizando uma área total amostrada de $25.920 \mathrm{~m}^{2}$, distribuídas de forma aleatória na área, por ser tratar de um inventário por "Amostragem Casual Simples".

\section{Dados Coletados}

Todos os indivíduos encontrados nas 36 parcelas tiveram sua circunferência à altura do peito (CAP), mensuradas com o uso de uma fita métrica, e $20 \%$ destes indivíduos mais os indivíduos considerados dominantes tiveram sua altura total (HT) coletada com o hipsômetro "clinômetro 360”․ As demais árvores tiveram suas alturas determinadas através da aplicação de uma equação hipsométrica ajustada para cada parcela.

De posse de todos os dados, foi realizada a distribuição diamétrica da população para nortear o procedimento de cubagem rigorosa. Scolforo e Figueiredo (1998) propõe que para cada classe diamétrica encontrada, seja cubado um determinado número de árvores para compor a 
amostra piloto do procedimento de cubagem rigorosa, e posteriormente realize se o cálculo e recálculo da intensidade amostral com os dados da amostra piloto para determinar o número de árvores da amostra definitiva do procedimento, de forma a obter um erro abaixo de $10 \%$.

O método de cubagem rigorosa utilizado foi o método de Newton, e no total foram cubadas 70 árvores de diferentes classes de diâmetro.

\section{Tratamentos}

Para testar as diferentes metodologias de determinação do volume de madeira em pé utilizou-se um Delineamento em Blocos Casualizados (DBC) constituído por três tratamentos (diferentes metodologias, descritas abaixo) com trinta e seis repetições (parcelas utilizadas no inventário florestal).

Tratamento 01: Volume individual de todas as árvores mensuradas no inventário florestal, determinados através da equação: Volume = área seccional x Altura x Fator de Forma Médio. $\mathrm{O}$ Fator de Forma Médio foi calculado através do calculo da média dos Fatores de Forma encontrados para cada árvore cubada. Fator de forma: razão entre o volume real e o volume cilíndrico de uma árvore.

Tratamento 02: Volume individual de todas as árvores mensuradas no inventário florestal, determinados através da equação: Volume = área seccional $\times$ Altura $\times$ Fator Razão. Fator Razão (Fr): razão entre o somatório dos volumes reais e somatório dos volumes cilíndricos de todas as árvores cubadas.

Tratamento 03: Volume individual de todas as árvores mensuradas no inventário florestal, determinados através da aplicação da Equação Volumétrica selecionada após o ajuste de seis modelos volumétricos selecionados na literatura através da ferramenta de análise de regressão do software Excel, segundo os valores do coeficiente de determinação, coeficiente de correlação e erro padrão de estimativa encontrados para cada equação ajustada com os dados da cubagem rigorosa.

\section{Avaliações}

Os valores obtidos pela aplicação dos diferentes tratamentos para as árvores existentes nas 36 parcelas do inventário florestal foram submetidos ao programa estatístico denominado ASSISTAT Versão 7.7 beta (Silva e Azevedo, 2002), onde foi utilizado o procedimento de análise de variância e Teste de Média de Scott knott a 5\% de probabilidade para realizar a comparação entre os tratamentos. 
RESULTADOS

De posse de todos os dados coletados nas 36 parcelas do inventário florestal aplicamos separadamente o Fator de Forma Médio encontrado que foi de 0,50 (1), o Fator Razão que foi de 0,48 (2) e a Equação Volumétrica gerada pelo modelo de Spur (3), que foi o modelo que gerou a melhor equação volumétrica segundo análise dos critérios de precisão estatísticos encontrados para os modelos ajustados com os dados obtidos na cubagem rigorosa das 70 árvores utilizadas neste trabalho.

Aplicado os tratamentos determinou se o volume sólido de madeira em pé existente por parcela, somando-se os volumes sólidos individuais, Tabela 1.

Tabela 1. Volumes obtidos por parcela após aplicação das diferentes metodologias de determinação do volume de madeira em pé.

\begin{tabular}{|c|c|c|c|c|c|c|c|}
\hline \multirow{2}{*}{ Parcela } & \multicolumn{3}{|c|}{ Tratamentos } & \multirow{2}{*}{ Parcela } & \multicolumn{3}{|c|}{ Tratamentos } \\
\hline & 1 & 2 & 3 & & 1 & 2 & 3 \\
\hline 1 & 11,5 & 11,0 & 10,8 & 19 & 9,4 & 9,0 & 8,8 \\
\hline 2 & 11,0 & 10,5 & 10,2 & 20 & 8,5 & 8,2 & 8,1 \\
\hline 3 & 9,9 & 9,5 & 9,3 & 21 & 7,7 & 7,4 & 7,3 \\
\hline 4 & 9,0 & 8,7 & 8,6 & 22 & 8,1 & 7,8 & 7,6 \\
\hline 5 & 9,3 & 8,9 & 8,8 & 23 & 11,9 & 11,4 & 11,1 \\
\hline 6 & 10,2 & 9,8 & 9,7 & 24 & 10,6 & 10,2 & 10,0 \\
\hline 7 & 12,0 & 11,5 & 11,2 & 25 & 11,9 & 11,4 & 11,1 \\
\hline 8 & 13,2 & 12,7 & 12,3 & 26 & 10,7 & 10,2 & 10,0 \\
\hline 9 & 8,7 & 8,4 & 8,2 & 27 & 11,4 & 11,0 & 10,7 \\
\hline 10 & 11,3 & 10,8 & 10,6 & 28 & 12,5 & 12,0 & 11,6 \\
\hline 11 & 11,3 & 10,9 & 10,6 & 29 & 13,5 & 12,9 & 12,5 \\
\hline 12 & 12,2 & 11,7 & 11,4 & 30 & 12,8 & 12,3 & 12,0 \\
\hline 13 & 9,2 & 8,8 & 8,6 & 31 & 11,9 & 11,4 & 11,1 \\
\hline 14 & 10,6 & 10,2 & 9,9 & 32 & 12,7 & 12,2 & 11,8 \\
\hline 15 & 9,0 & 8,6 & 8,5 & 33 & 12,4 & 11,9 & 11,5 \\
\hline 16 & 9,7 & 9,3 & 9,1 & 34 & 9,3 & 9,0 & 8,7 \\
\hline 17 & 10,8 & 10,4 & 10,1 & 35 & 10,3 & 9,9 & 9,7 \\
\hline 18 & 10,2 & 9,8 & 9,6 & 36 & 10,9 & 10,5 & 10,2 \\
\hline
\end{tabular}

Submetidos os dados as análises, constatamos a existência de diferença significativa entre os tratamentos, conforme Tabela 2. 
Tabela 2. Análise de Variância para a FV Tratamento

\begin{tabular}{ccccc}
\hline FV & GL & SQ & QM & F \\
\hline Blocos & 35 & 217,53594 & 6,21531 & $1101,4546^{* *}$ \\
Tratamentos & 2 & 8,28705 & 4,14352 & $734,2998^{* *}$ \\
Resíduo & 70 & 0,39500 & 0,00564 & \\
\hline Total & 107 & 226,21798 & & \\
\hline CV $(\%)=0,73$ & Média Geral $=10,34688$ & \multicolumn{2}{c}{ Ponto Médio $=10,40244$} \\
\hline
\end{tabular}

** Significativo ao nível de $1 \%$ de probabilidade $(p<.01)$; Significativo ao nível de $5 \%$ de probabilidade ( $p<.05)$; ns não significativo $(p>=.05)$

A diferença entre os tratamentos encontra se na Tabela 3.

Tabela 3 - Teste de Média Scott - knott para a FV Tratamento, nível de significância de 0,05.

\begin{tabular}{cc}
\hline Tratamento & Média \\
\hline 1 & $10.71301 \mathrm{a}$ \\
2 & $10.28449 \mathrm{~b}$ \\
3 & $10.04315 \mathrm{c}$ \\
\hline
\end{tabular}

Obs.: médias seguidas pelas mesmas letras são consideradas estatisticamente iguais, através do teste Scott-Knott ( $p$ <0.05). Descrição dos tratamentos: T1 - Forma de Forma, T2 - Fator Razão, e T3 - Equação Volumétrica.

Além das comparações, realizou se o processamento do inventário florestal definitivo com os volumes das 36 parcelas obtidos pelas diferentes metodologias:

Tabela 4 - Resultados do Inventário Florestal por diferentes métodos de determinação do volume de madeira em pé.

\begin{tabular}{|c|c|c|c|c|c|c|c|c|c|}
\hline \multirow{3}{*}{ Trat. } & \multirow{3}{*}{ I.A. } & \multirow{3}{*}{$\begin{array}{c}\text { Erro Abs. } \\
\left(\mathrm{m}^{3} \cdot \text { parcela }^{-1)}\right.\end{array}$} & \multirow{3}{*}{$\begin{array}{l}\text { Erro } \\
(\%)\end{array}$} & \multicolumn{6}{|c|}{ Intervalo de Confiança $\left(\mathrm{m}^{3}\right)$} \\
\hline & & & & \multicolumn{2}{|c|}{ Parcela } & \multicolumn{2}{|c|}{ Hectare } & \multicolumn{2}{|c|}{ População } \\
\hline & & & & Inf. & Sup. & Inf. & Sup. & Inf. & Sup. \\
\hline 1 & 10 & 0,51 & 4,75 & 10,2 & 11,2 & 141,7 & 155,9 & 77779,4 & 85534,5 \\
\hline 2 & 10 & 0,49 & 4,75 & 9,8 & 10,8 & 136,1 & 149,6 & 74668,2 & 82113,1 \\
\hline 3 & 10 & 0,46 & 4,59 & 9,6 & 10,5 & 133,1 & 145,9 & 73040,0 & 80062,0 \\
\hline
\end{tabular}

Onde: Trat. = Tratamento, I.A. = Intensidade amostral, Inf. = Limite Inferior, e Sup. = Limite Superior.

\section{DISCUSSÕES}

Os tratamentos que apresentaram as maiores médias foram os métodos de determinação do Fator de Forma Médio e Fator Razão, Tratamento 1 e 2, respectivamente. 
Segundo Couto e Bastos (1987) o uso de metodologias que trabalham com o produto do volume cilíndrico por uma constante que represente a forma da árvore, como o fator de forma médio, é menos preciso que o uso de equações volumétricas; e por isso deve ser restrito às condições locais de sítio. Qualquer extrapolação além desses limites pode ser perigosa sob o ponto de vista de previsão dos resultados finais (COUTO; BASTOS, 1987).

O Método de ajuste de modelos volumétricos é o mais preciso dos métodos de determinação de volume de árvores em pé por utilizar o cálculo de volume sólido, árvore a árvore, através de modelos matemáticos, especialmente testados para apresentar os menores erros possíveis (COUTO; BASTOS, 1987).

De acordo com Guimarães e Leite (1996) citado por Azevedo et al. (2011), o emprego de equações volumétricas constitui o procedimento mais eficiente para a quantificação da produção em volume de um povoamento florestal. Segundo Machado, et al. (2006) o emprego de equações volumétricas é o procedimento de maior uso na estimativa do volume individual de árvores.

Apesar dos bons resultados que comprovadamente são obtidos na estimativa do volume de madeira através de equações o seu uso muitas vezes requer softwares específicos e conhecimentos em regressão, para ajustar os diferentes modelos volumétricos e para selecionar a melhor equação volumétrica.

Thomas et al. (2006) ao ajustar 09 modelos volumétricos para determinação do volume com casca partindo de dados de cubagem de um povoamento de Pinus taeda encontrou bom desempenho estatístico para todos os modelos quanto aos valores de coeficiente de determinação, variando de 0,9388 a 0,9929; demonstrando um alto grau de ajuste de descrição da variável dependente, volume, pelas variáveis independentes, dap e altura.

Os resultados do coeficiente de determinação de modelos volumétricos para plantações florestais encontrados em outros trabalhos quase sempre se situam próximos de 0,9 , como em Santana et al. (2005) citado por Azevedo et al. (2011), no qual foi testado o modelo de Schumacher-Hall com algumas transformações para um plantio de Eucalyptus grandis no estado de São Paulo. Das seis equações testadas por Azevedo et al. (2011), a que apresentou melhor ajuste, tanto para o volume com casca como para o sem casca, foi a equação de Schumacher-Hall, apresentando maior coeficiente de determinação ajustado $(0,8745$ e 0,8737).

Segundo Rolim et al. (2006) citado por Miguel e Leal (2012), os modelos de simples entrada de Kopezky- Gehradt e de Hohenald-Krenn são tendenciosos. Miguel e Leal (2012) identificaram para os modelos de Kopezky-Gehrhardt, e Dissescu-Meyer, uma clara tendência de subestimativa 
para as árvores de menores diâmetros, enquanto que o modelo de Hohenadl-Krenm apresentou ligeira tendência em superestimativa para estas mesmas árvores de menores diâmetros.

Segundo Guimarães (1986) a seleção de uma equação para estimativa de volume deve satisfazer a dois critérios, ou seja, estimar os dados dentro dos limites de precisão requeridos e ser caracterizada pela facilidade de uso. De modo geral, os resultados conduzem a alguns modelos tradicionalmente conhecidos. Quando as variáveis sofrem transformações para a forma logarítmica, normalmente o modelo de melhor ajuste é o proposto por Schumacher - Hall (1933); quando na forma linear, a combinação das variáveis diâmetro e altura, proposta por Spurr (1952) tende a ser mais explicativa do volume (GUIMARÃES, 1986).

\section{CONCLUSÕES}

Entre as metodologias testadas houve diferença significativa; entretanto a literatura, Couto e Bastos (1987), trás que a melhor metodologia e mais utilizada é a de equações volumétricas, dentre os modelos volumétricos os melhores são o de dupla entrada, que envolve Diâmetro e Altura;

Muitos modelos matemáticos foram desenvolvidos para estimar o volume de povoamentos florestais, e apesar da eficiência de alguns modelos, estes nem sempre se ajustam a todas as espécies e condições, sendo recomendável testá-los e, por meio de estatísticas adequadas, identificar o melhor para cada caso; recomendamos, como Guimarães (1986), apenas os modelos Tradicionais de Spurr e Schumacher-Hall para serem ajustados e comparados.

$\mathrm{Na}$ seleção do melhor modelo deve-se observar os resultados do coeficiente de determinação que deve estar o mais próximo de 0,9.

Após processar o Inventário Florestal todas as metodologias utilizadas neste trabalho apresentaram Erro abaixo do permitido para florestas plantadas que é de $10 \%$.

\section{REFERÊNCIAS}

AZEVEDO, T. L.; MELLO, A. A. de; FERREIRA, R. A.; SANQUeTTA, C. R.; NAKAJIMA, N. Y. Equações hipsométricas e volumétricas para um povoamento de Eucalyptus sp. localizado na FLONA do Ibura, Sergipe. Agrária: Revista Brasileira de Ciências Agrárias. v.6, n.1., p.105-112, 2011. http://dx.doi.org/10.5039/agraria.v6i1a861

COUTO, H. T. Z.; BASTOS, N. L. M.. Modelos de Equações de Volume e Relações Hipsométricas para Plantações de Eucalyptus no Estado de São Paulo. IPEF, n.37, p.33-44, 1987.

GUIMARÃES, D. P. Variação do modelo de Schumacher - Hall para ajuste de equações volumétricas. Planaltina, EMBRAPA - CPAC, 1986. 
GUIMARÃES, D. P.; LEITE, H. G. Influência do número de árvores na determinação de equação volumétrica para Eucalyptus grandis. Scientia Forestalis, Piracicaba, n. 50, p.37-42, 1996.

MACHADO, S. A.; FIGUEIREDO FILHO, A. Dendrometria. Guarapuava: Unicentro. 2 Ed., 2006.

MIGUEL, E. P.; LEAL, F. A. Seleção de Equações Volumétricas para a predição do volume total de Eucalyptus urophylla S. T. Blake na Região Norte do Estado de Goiás. Enciclopédia Biosfera, Centro Científico Conhecer. Goiânia, v.8, N.14, p.1372, 2012.

ROLIM, S. G.; COUTO; H. T. Z.; JESUS, R. M.; FRANÇA, J. T. Modelos volumétricos para a Floresta Nacional do Tapirapé-Aquirí, Serra dos Carajás (PA). Acta Amazônica, Amazônia, v.36, p.107-114, 2006.

SANTANA, C.; MELlO, A. A. de; EISFELD, R. de L.; SANQUETA, C. R. Sistema de equações para simulação do crescimento e da produção em povoamentos de Eucalyptus grandis Hill Ex Maiden. sem desbaste baseado no modelo de clutter. Revista do Centro de Ciências Agrárias e Ambientais, Paraná, v.1, n.2, p.239-256, 2005.

SCHUMACHER, F. X.; HALL, F. S. Logarithmic expression of tree volume. Journal of Agricultural Research, 47:719-34, 1933.

SCOLFORO, J. R. S.; FILHO, A. F. Biometria florestal: medição e volumetria de árvores. Gráfica e Editora: UFLA/FAEPE, 1998.

SILVA, F. de A. S.; AZEVEDO, C. A. V. de. Versão do programa computacional Assistat para o sistema operacional Windows. Revista Brasileira de Produtos Agroindustriais, Campina Grande, v. 4, n. 1, p.71-78, 2002.

SPURR, S.H. Forest Inventory. New York, The Ronald Press, 1952. 476p.

THOMAS, C.; ANDRADE, C. M.; SCHNEIDER, P. R.; FINGER, C. A. G. Comparação de equações volumétricas ajustadas com dados de cubagem e análise de tronco. Ciência Florestal, Santa Maria, v.16, n.3, p.319-327, 2006. 